\title{
Profilassi e gestione delle recidive della calcolosi renale
}

Commento all'articolo: Medical management to prevent recurrent nephrolithiasis in adults: a systematic review for an American College of Physicians Clinical Guideline

\section{Paolo Beltrami, Filiberto Zattoni}

Clinica Urologica, Dipartimento di Scienze Chirurgiche, Oncologiche e Gastroenterologiche, Università degli Studi di Padova, Padova

In questo lavoro vengono analizzati i risultati presenti in letteratura riguardo i rischi e i benefici del trattamento profilattico della calcolosi renale recidivante. Con metodo statistico, che analizza trial randomizzati e controllati, gli Autori hanno cercato di dare una serie di risposte a domande riguardanti i pazienti affetti da nefrolitiasi recidivante, in merito ai risultati delle varie opzioni dietetiche e terapeutiche attualmente prescritte e agli eventuali effetti avversi (1).

Come segnalato dagli Autori i limiti dell'analisi sono rappresentati dal ridotto numero di studi impostati con un corretto disegno statistico e con degli endpoint adeguati. Molti si basano su analisi retrospettive di questionari riguardanti le abitudini alimentari somministrati a coorti di pazienti arruolati per altri studi. Le casistiche inoltre non sono uniformi né per quanto riguarda la composizione chimica dei calcoli né per l'analisi delle recidive (recidiva sintomatica o recidiva all'imaging).

I fattori alimentari litogeni sono molteplici e talvolta difficili da analizzare singolarmente, considerata la varietà di alimenti e la composizione chimica degli stessi. A ciò si aggiunge il diverso assorbimento dei singoli fattori, sia per motivi individuali che per la presenza di altre sostanze alimentari che lo possono condizionare. Indipendentemente dal regime alimentare poi, moltissimi altri fattori sono responsabili della litogenesi. Tra questi gli inibitori della cristallizzazione noti e non, non sempre facilmente dosabili e identificabili, la cui presenza o riduzione nel singolo individuo possono essere concausa di un aumentato rischio di recidiva.

Tutto ciò potrebbe essere uno dei tanti motivi dei variabili risultati presenti in letteratura riguardo i diversi regimi alimentari. Frequentemente infatti si trovano dati discordanti come, ad esempio, il fatto che l'assunzione di bibite acidificate con acido fosforico possa ridurre il rischio di recidiva litiasica secondo alcuni Autori (2), mentre ne aumenti il rischio secondo altri che ritengono l'acido ortofosforico un potenziale agente litogeno (3). Il tè, bevanda ricca di ossalati, da alcuni viene ritenuto litogeno, mentre per altri il potere diuretico indotto dalla caffeina contenuta nello stesso lo rende una bevanda in grado di ridurre l'incidenza di recidive litiasiche (4).

Quello che di certo emerge riguardo l'alimentazione è che l'idratazione costituisce il fulcro di qualsiasi atteggiamento dietetico per ridurre il rischio di recidiva. Anche la modalità di assunzione e la qualità delle acque possono avere un ruolo nella profilassi. È consigliabile infatti impiegare acque oligominerali, a basso contenuto di calcio e di sodio, e di assumere liquidi durante tutto l'arco della giornata e in quantità tale da mantenere una diuresi uniforme e superiore a 2 litri/die. Da evitare i carichi idrici rapidi e i periodi seppur brevi di disidratazione, come si verifica durante la stagione estiva o in corso di attività fisica intensa.

Un altro fattore da tenere in considerazione, anche se non facilmente analizzabile, riguarda la morfologia e la funzionalità della via escretrice. È noto che frequentemente le recidive litiasiche si localizzano dallo stesso lato e nella stessa sede. Esistono pertanto fattori predisponenti a livello locale (placche di Randall, conformazione anatomica e anomala peristalsi della via escretrice) $(5,6)$ che possono coesistere con le alterazioni dei metaboliti urinari, tanto da sbilanciare l'equilibrio idrosalino e facilitare la precipitazione di cristalli.

Per quanto riguarda la profilassi farmacologica, tiazidi, citrati e allopurinolo evidenziano una riduzione del rischio di recidiva nei pazienti trattati rispetto al placebo, con una limitata comparsa di effetti collaterali. I risultati variabili potrebbero anche dipendere dalla composizione chimica dei calcoli che nelle varie serie non sempre è stata analizzata in maniera approfondita.

In ogni caso, in questo lavoro, l'analisi dei risultati degli studi presenti in letteratura è molto accurata ed ampia ed è senza dubbio da tenere in considerazione nel programma di follow-up del paziente affetto da nefrolitiasi. Riteniamo tuttavia che la profilassi idropinica, alimentare e farmacologica debba essere affiancata a un monitoraggio di imaging frequente, in modo da evidenziare la comparsa di recidive, quando queste siano di dimensioni ridotte: ciò consente un trattamento endourologico retrogrado con ottimi risultati prima che le dimensioni del calcolo raggiungano valori tali da richiedere trattamenti più invasivi.

Dichiarazione di conflitto di interesse: Gli Autori dichiarano di non avere conflitto di interessi.

Contributi economici agli Autori: Gli Autori dichiarano di non avere ricevuto sponsorizzazioni economiche per la preparazione dell'articolo. 
Indirizzo degli Autori:

Dr. Paolo Beltrami

Clinica Urologica, Dipartimento di Scienze Chirurgiche,

Oncologiche e Gastroenterologiche

Università degli Studi di Padova

Via Giustiniani 2

35128 Padova

paolo.beltrami@sanita.padova.it

\section{Bibliografia}

1. Fink HA, Wilt TJ, Eidman KE, et al. Medical management to prevention recurrent nephrolithiasis in adults: a systematic review for an American College of Physicians. Clinical guideline. Ann Intern Med 2013; 158(7): 535-43.

2. Shuster J, Jenkins A, Logan C, et al. Soft drink consumption and urinary stone recurrence: a randomized prevention trial. J Clin Epidemiol 1992; 45: 911-6.

3. Eisner BH, Asplin JR, Goldfarb DS, Ahmad A, Stoller ML. Citrate, malate and alkali content in commonly consumed diet sodas: Implications for nephrolithiasis treatment. J Urol 2010; 183: 2419-23.

4. Ferraro PM, Taylor EN, Gambaro G, Curhan GC. Soda and other beverages and the risk of kidney stones. Clin J Am Soc Nephrol 2013; 8: 1389-95.

5. Strohmaier WL, Hörmann M, Schubert G. Papillary calcifications: a new prognostic factor in idiopathic calcium oxalate urolithiasis. Urolithiasis 2013; 41: 475-9.

6. Ruggera L, Gambaro G, Beltrami P, Martignoni G, Zattoni F. Percutaneous and transureteral biopsies of renal papillae: safe and appropriate procedures for in vivo histologic analysis in stone formers. J Endourol 2011; 25: 25-30. 